\title{
Assessing Cognitive Performance in Badminton Players: A Reproducibility and Validity Study
}

\author{
by \\ Tanja van de Water ${ }^{1}$, Barbara Huijgen ${ }^{1}$, Irene Faber ${ }^{2,3,4}$, Marije Elferink-Gemser ${ }^{1}$
}

Fast reaction and good inhibitory control are associated with elite sports performance. To evaluate the reproducibility and validity of a newly developed Badminton Reaction Inhibition Test (BRIT), fifteen elite ( $25 \pm 4$ years) and nine non-elite (24 \pm 4 years) Dutch male badminton players participated in the study. The BRIT measured four components: domain-general reaction time, badminton-specific reaction time, domain-general inhibitory control and badminton-specific inhibitory control. Five participants were retested within three weeks on the badminton-specific components. Reproducibility was acceptable for badminton-specific reaction time (ICC $=0.626, C V=6 \%$ ) and for badminton-specific inhibitory control (ICC $=0.317, C V=13 \%$ ). Good construct validity was shown for badmintonspecific reaction time discriminating between elite and non-elite players $(F=6.650, p<0.05)$. Elite players did not outscore non-elite players on domain-general reaction time nor on both components of inhibitory control $(p>0.05)$. Concurrent validity for domain-general reaction time was good, as it was associated with a national ranking for elite ( $\rho$ $=0.70, p<0.01)$ and non-elite $(\rho=0.70, p<0.05)$ players. No relationship was found between the national ranking and badminton-specific reaction time, nor both components of inhibitory control $(p>0.05)$. In conclusion, reproducibility and validity of inhibitory control assessment was not confirmed, however, the BRIT appears a reproducible and valid measure of reaction time in badminton players. Reaction time measured with the BRIT may provide input for training programs aiming to improve badminton players' performance.

Key words: racquet sports, processing speed, reaction time, inhibitory control, elite athletes.

\section{Introduction}

Badminton is an interceptive, fast racket sport in terms of shuttle velocity with an average velocity reaching up to $70 \mathrm{~m} / \mathrm{s}$ (Phomsoupha and Laffaye, 2014, 2015). On average six strokes are played during rallies of seven seconds and the average stroke frequency is high with approximately one shot per second (Phomsoupha and Laffaye, 2015). To keep up with the high game speed, a fast reaction time is utmost important to achieve elite performance (Loureiro and Freitas, 2012). The unpredictability of the opponent's actions and the usage of feint strokes in elite badminton indicate inhibitory control as crucial for elite performance as well. Although it is recognised that a combination of excellence in technical, tactical, anthropometrical, physical and mental skills is needed for elite sports performance (Elferink-Gemser et al., 2011), fast reaction time and outstanding inhibitory control are suggested to be indispensable for expertise in

\footnotetext{
1 - Center for Human Movement Sciences, University Medical Centre Groningen, University of Groningen, Groningen, The Netherlands.

2 - Faculty of Physical Activity and Health, Saxion University of Applied Sciences, Enschede, The Netherlands.

3 - Radboud University Medical Centre, Radboud Institute for Health Sciences, IQhealthcare, Nijmegen, The Netherlands.

4 - Sports Science and Medical Committee, International Table Tennis Federation, Lausanne, Switzerland.
} 
badminton. However, due to limited available literature, the importance of reaction time and inhibitory control to reach and perform on the elite level in badminton can only be speculated.

Reaction time is a measure of processing speed and reflects response efficiency in information processing tasks. A fast reaction time is thought to be necessary for quick and accurate reactions in fast paced sports (Voss et al., 2010). Reaction time is considered a lower-level cognitive function that supports basic information processing (Wetherell, 1997). Inhibitory control refers to the ability to stop a planned or dominant motor response and is a higher-level cognitive function (Williams et al., 1999). Higher-level cognitive functions are often called executive functions and are important in tasks that demand among others concentration, coordination, change and overriding a strong internal or external pull (Diamond, 2006).

The relation between lower-level cognitive performance and sports performance is described in the reviews of Voss and Kramer (2010) and Mann et al. (2007). The review of Voss and Kramer (2010) examined domain-general reaction time by means of the component skills approach, that measures cognitive performance in a context outside sports (Alves et al., 2013). Mann et al. (2007) examined context-specific reaction time and included studies that applied the expert performance approach. This approach makes use of a test-setting that reflects the demands of a specific sport. Despite of the different approach, both reviews showed that a fast reaction time was important for elite performance, especially in interceptive sports like badminton (Mann et al., 2007; Voss et al., 2010). This suggests that elite badminton players are able to react faster than non-elite badminton players.

Recent studies focusing on higher-level cognitive performance in soccer revealed that elite or talented soccer players outscored non-elite players on domain-general inhibitory control tasks (Huijgen et al., 2015; Verburgh et al., 2014; Vestberg et al., 2012). Kida et al. (2005) assessed the context-specific inhibitory control of baseball players and were not only able to discriminate elite players from non-elite, but also from subelite ones. In summary, measures of domaingeneral and context-specific inhibitory control seem to be able to distinguish players from different performance levels (Huijgen et al., 2015; Kida et al., 2005; Verburgh et al., 2014; Vestberg et al., 2012).

Up to now, literature on reaction time and inhibitory control in badminton players of various performance levels has been limited. As an exception, Loureiro and Freitas (2012) presented that elite badminton players had a significantly faster badminton-specific reaction time than nonelite players. However, this study provided no evaluation of reproducibility and validity of the used test and did not include the assessment of inhibitory control. For an accurate examination of reaction time and inhibitory control in badminton players of various performance levels, both domain-general and badminton-specific reaction time as well as inhibitory control should be assessed. Considering that, to our knowledge, no such a test existed (Faber et al., 2016), a test was developed measuring four components: domaingeneral reaction time, badminton-specific reaction time, domain-general inhibitory control and badminton-specific inhibitory control. For the assessment of domain-general reaction time and inhibitory control, a procedure similar to the Stop Signal Task was included in the test (Williams et al., 1999). The 'stop signal task' is a reproducible measure of domain-general reaction time (splithalf reliability $=0.97$ ) and inhibitory control (splithalf reliability $=0.91$, ICC $=0.86$ ) (Congdon et al., 2012; Williams et al., 1999). Badminton-specific reaction time and inhibitory control were assessed in a procedure based on the badminton-specific test used by Loureiro and Freitas (2012), completed with badminton-specific match play characteristics. These badminton-specific characteristics include reaching movements of the arm to simulate stroke execution, a semi-squat central position (Loureiro and Freitas, 2012), rapid whole body movements to the forehand and backhand side (i.e. lunges) (Hong et al., 2014; Kuntze et al., 2010) and visual cues (Lees, 2003; Phomsoupha and Laffaye, 2015).

The aim of the current study was to evaluate the newly developed test on 1) the reproducibility for the assessment of badmintonspecific reaction time and inhibitory control, and 2) the validity for the assessment of all four components of the test, and as such containing both domain-general and badminton-specific reaction time as well as inhibitory control in elite 
and non-elite badminton players. Validity was investigated by comparing the test results of elite badminton players with non-elite badminton players and by examining the relationship between the test results and the national ranking for elite and non-elite players, separately. Reproducibility of the badminton specific part was hypothesized to be at an acceptable level, since the procedures were similar to those used in the 'stop signal task'. Moreover, regarding the evaluation of validity, it was hypothesized that elite badminton players would outscore non-elite players on measures of reaction time and inhibitory control and that there would be moderate relationships between reaction time and the national ranking as well as between inhibitory control and the national ranking.

\section{Material and Methods}

\section{Participants}

In total 24 Dutch male badminton players divided into two groups participated in the study. The elite group $(\mathrm{n}=15 ; 25 \pm 4$ years $)$ included male badminton players who took part in the Dutch national competition in the season of 20152016 and were ranked within the top 100 on the Dutch national men's singles badminton ranking list at the moment of test execution. The non-elite group ( $\mathrm{n}=9 ; 24 \pm 4$ years) included players who participated in the Dutch regional competition in one of the nine regions in the season of 2015-2016 and had a ranking exceeding 100 on the Dutch national men's singles badminton ranking list at the moment of test execution. The characteristics of the elite and non-elite groups are presented in Table 1 . Elite players trained significantly more hours per week $(p<0.001)$ and had a higher ranking $(p<0.001)$ than non-elite players.

\section{Study design}

A two-fold design was used to evaluate the reproducibility and validity of the test. First, the reproducibility of the assessment of badminton-specific reaction time and inhibitory control was examined by means of a test-retest design. The time between the initial test and retest ranged from two to three weeks. In the second part of the study, the validity of all four components of the test was evaluated. The test was examined on its ability to discriminate between elite and non-elite players and on the relationship between test results and the national ranking.

Measures

The newly developed Badminton Reaction Inhibition Test (BRIT) consisted of four components: domain-general reaction time, badminton-specific reaction time, domain-general inhibitory control and badminton-specific inhibitory control. The domain-general components were measured with the domaingeneral stop signal task, of which procedure is similar to the Stop Signal Task of Logan and Cowan (1984). The badminton-specific components were measured with the badmintonspecific stop signal task.

Domain-general stop signal task

The task involved go-trials and stop-trials. During a go-trial, a cartoon cyclist (go-stimulus) appeared for $1000 \mathrm{~ms}$ in the centre of the computer screen either facing the left or right side. The players had to respond to the go-stimulus as fast as possible by pressing a key on the keyboard with the spatially corresponding index finger. In $25 \%$ of all trials a red cross appeared at the former location of the go-stimulus after a certain stop signal delay (SSD). These were the so called stoptrials. Whenever the stop-stimulus was presented, the motor response should be stopped immediately (i.e. inhibitory control). The initial SSD was set at $175 \mathrm{~ms}$ and increased with $50 \mathrm{~ms}$ when the player succeeded to inhibit the response and decreased with $50 \mathrm{~ms}$ when the player failed to do so, resulting in a success rate around $50 \%$ to inhibit the response (Verbruggen and Logan, 2009). In every trial a fixation point preceded the go-stimulus for $500 \mathrm{~ms}$ and the inter-trial delay was $1000 \mathrm{~ms}$.

The task consisted of two practice blocks and four experimental blocks. The first practice block included 16 go-trials and no stop-trials and the second practice block included 32 trials with $25 \%$ of stop-trials. After the two practice blocks, four test blocks of 32 trials with $25 \%$ of stop-trials followed. Reaction time was the time in milliseconds between go-stimulus appearance and pushing of the corresponding key during gotrials. The following outcome variables were generated: DGRT (Domain General Reaction Time) which was the average reaction time during the four test blocks and DGIC (Domain General Inhibitory Control) which was calculated by subtracting the mean SSD from the DGRT. 
Reaction times faster than $200 \mathrm{~ms}$ or slower than $1000 \mathrm{~ms}$ were referred to as anticipation errors or omission errors and were not included in the analysis (Kida et al., 2005). For the Stop Signal Test, high reliability for domain general reaction time and domain general inhibitory control had been reported (split-half reliability $=0.91$ and 0.93 , respectively) (Williams et al., 1999).

Badminton-specific stop signal task

The badminton-specific stop signal task made use of the fitLight Trainer ${ }^{\mathrm{TM}}$ (fitLight Sports Corp., Canada). The fitLight Trainer ${ }^{\mathrm{TM}}$ is a wireless reaction training system consisting of LED powered lights controlled by a tablet. The lights can be deactivated by touch. During the test, the players were positioned in front of a wall on which three lights of the fitLight Trainer ${ }^{\mathrm{TM}}$ were placed as depicted in Picture 1. The main task was to deactivate the correct light as fast as possible as soon as it lit up. The following features were added to resemble badminton match play: 1) all lights had to be deactivated with the players' preferred hand (the hand in which players normally hold their racket), 2) the horizontal distance between the centre light and the two outer lights was $130 \mathrm{~cm}$ and therefore afforded the use of a small lunge to either the forehand side (side of the preferred hand) or the backhand side (side opposite to the preferred hand) to reach them (Hong et al., 2014; Kuntze et al., 2010) and 3) the lights were placed at a height of $110 \mathrm{~cm}$, affording the players to bent through their knees to deactivate the lights in an easy manner and this position reflected the central position during badminton play (Loureiro and Freitas, 2012).

The task included go-trials and stop-trials. During a go-trial, the centre light lit up (yellow or pink) and it had to be deactivated, directly followed by deactivating the corresponding outer light; the yellow light corresponded with the left outer light and the pink light with the right outer light. After deactivating the outer light, the player returned to the central position and another trial started. A stop-trial was identical to a go-trial, except that after deactivation of the centre light, all three lights turned red after a certain amount of time called the stop signal delay (SSD). As a consequence, the player was already moving from the recently deactivated centre light to the corresponding outer light once the lights turned red. The red lights reflected the stop-stimuli, indicating that the motor response had to be stopped immediately and the outer light should not be deactivated. After correctly inhibiting the motor response or (by error) deactivating the outer light, the player returned to the central position again. All blocks consisted of 12 trials. The time between centre light deactivation and appearance of the next trial was set at $2150 \mathrm{~ms}$ for all trials and before each trial the centre light turned blue for $500 \mathrm{~ms}$ as a fixation stimulus.

The test consisted of two conditions presented in the following order: the go condition and the go-stop condition. Both conditions started with one practice block followed by the test block(s). The go condition was comprised of merely go-trials and included one test block, while the go-stop condition included go-trials and $25-33 \%$ of stop-trials per block. During the practise block, the SSD was set at $100 \mathrm{~ms}$ and the players were told that the red lights were only a visual distraction and that they should still react as fast as possible to get used to the stop-stimuli (Williams et al., 1999). All players started with the first test block characterised by a stop signal delay (SSD) of $100 \mathrm{~ms}$. Whenever the success rate (\% of correctly inhibited stop-trials in the current block) was $50 \%$ or higher, the following block consisted of a SSD increased by $50 \mathrm{~ms}$. Whenever the success rate was lower than $50 \%$, a second test block with the same SSD was performed. The test finished when two consecutive blocks with a success rate lower than 50\% appeared. The SSD of these last two blocks was called the End SSD.

Reaction time was determined as the time in milliseconds between deactivation of the centre light and deactivation of the outer light. Go BSRT (Badminton Specific Reaction Time of the Go condition) and Go-stop BSRT (Badminton Specific Reaction Time of the Go-stop condition) represented the average reaction time during gotrials for the go and go-stop condition, respectively. This was further subdivided into Go/Go-stop forehand BSRT (BSRT to the side of the players' preferred hand) and go/go-stop backhand BSRT (BSRT to the side opposite to the players' preferred hand). BSIC (Badminton Specific Inhibitory Control) was calculated by subtracting the End SSD from the Go-stop BSRT. Shorter BSIC reflected better inhibitory control. Reaction times faster than $200 \mathrm{~ms}$ or slower than $1000 \mathrm{~ms}$ were referred to as anticipation error or 
omission error and were not included in the analysis (Kida et al., 2005).

\section{Procedures}

The study was conducted in compliance with the Declaration of Helsinki and approved by the Ethics Committee of the Medical Faculty of the University of Groningen (January, 2016). Written informed consent was obtained prior to testing.

Testing was conducted under supervision of one researcher with help of two other experienced test leaders and took place during the Dutch National Championships or prior to a training session. During the testing session, the players executed the BRIT and filled in a questionnaire about badminton related activities over the past eight years, including hours of training per week and starting age. Not all players completed all test components of the BRIT and the questionnaire due to limited time or unwillingness to do a physically demanding test (badminton-specific component BRIT) prior to match play.

\section{Statistical Analysis}

IBM SPSS Statistics 23 (IBM Corp. Somers, NY) was used for data analysis. All test variables were checked for normality by examining normality plots, z-scores of skewness and kurtosis. The ranking was considered as ordinal data. For both the elite and non-elite groups, the mean and standard deviation of every outcome variable was determined.

First, the reproducibility of badmintonspecific reaction time and inhibitory control during the go-stop condition (respectively go-stop BSRT and go-stop BSIC) was examined by measures of absolute and relative test-retest reliability and measures of agreement. Absolute reliability was examined by means of paired $\mathrm{t}$ tests and relative reliability by means of the intraclass correlation coefficient (ICC, model oneway random). An ICC lower than .40 reflected poor reliability, between .40 and .75 fair to good reliability and above .75 excellent reliability (Fleiss, 1999). Measure of agreement was determined by the coefficient of variation (CV) (de Vet et al., 2006).

Second, the construct validity of all four components of the BRIT was evaluated. This was done by examining the between group difference (elite versus non-elite) for all test variables separately by means of a one-way analysis of variance, supported by the Cohen's d effect-size. Finally, concurrent validity was investigated by examining the relationship between the national ranking and all test variables. This was examined for the elite and non-elite players separately due to the spread in the ranking between the two participant groups. The Spearman's rho correlation coefficient supported by the coefficient of determination as an effect-size was calculated for examination of concurrent validity. Alpha was set at .05 for all analyses.

\section{Results}

\section{Reproducibility}

Reproducibility outcomes for badmintonspecific reaction time (BSRT) showed a mean difference between the initial test and retest of 1 ms (95\% CI: $-78-80)$ and met the criteria of good relative reliability (ICC $=0.626, p>0.05$ ) (Fleiss, 1999). The coefficient of variation (CV) for BSRT was $6 \%$. Badminton-specific inhibitory control (BSIC) showed a mean difference between the initial test and retest of $31 \mathrm{~ms}$ (95\% CI: $-47-110)$ and presented poor to fair relative reliability (ICC $=0.317, p>0.05$ ) (Fleiss, 1999). The CV for BSIC was $13 \%$.

\section{Construct validity}

The results of the construct validity analyses are presented in Table 2. Elite and nonelite players did not differ in domain-general reaction time (DGRT). Elite players outscored non-elite players on measures of badmintonspecific reaction time during the go condition (Go BSRT) and on measures of badminton-specific backhand reaction time during the go condition (Go backhand BSRT) $(p<0.05)$. No differences between elite and non-elite players were found for badminton-specific forehand reaction time during the go condition (Go forehand BSRT) nor for domain-general inhibitory control (DGIC) and badminton-specific inhibitory control (BSIC) $(p>$ 0.05).

\section{Concurrent validity}

Results on concurrent validity analyses are presented in Table 3 for the elite and non-elite players separately. The national ranking accounted for half of the variance in DGRT in elite and non-elite players. In Figure 1, the relationship between the national ranking and DGRT is depicted for both the elite and non-elite groups. 
No significant correlation was revealed between the ranking, BSRT and DGIC. The ranking was significantly negatively correlated with BSIC in non-elite players, but not in elite players.

Table 1

Characteristics of the elite and non-elite badminton players (mean $\pm S D$ )

\begin{tabular}{|c|c|c|c|c|c|c|c|}
\hline & $\mathbf{N}$ & Elite & $\mathbf{N}$ & Non-elite & $\mathbf{t}$ & df & $\begin{array}{l}p \text { (two- } \\
\text { tailed) }\end{array}$ \\
\hline Age (years) & 15 & $25 \pm 4$ & 9 & $24 \pm 4$ & 0.643 & 22 & 0.527 \\
\hline Body Height (cm) & 9 & $190 \pm 8$ & 8 & $185 \pm 3$ & 2.013 & 11 & 0.069 \\
\hline $\begin{array}{l}\text { Badminton training } \\
(\mathrm{h} / \mathrm{wk})\end{array}$ & 11 & $7 \pm 3$ & 7 & $2 \pm 1$ & 5.656 & 11 & $<0.001^{*}$ \\
\hline Starting age (years) & 11 & $8 \pm 2$ & 7 & $11 \pm 4$ & -1.912 & 16 & 0.074 \\
\hline Rankinga & 15 & $\begin{array}{c}39 \pm 24 \\
\text { Median }=35\end{array}$ & 9 & $\begin{array}{c}2753 \pm 1586 \\
\text { Median }=3184\end{array}$ & $\begin{array}{c}\mathrm{U}< \\
0.001^{\mathrm{a}}\end{array}$ & $Z=-4.50^{a}$ & $<0.001^{\mathrm{b}^{*}}$ \\
\hline
\end{tabular}

${ }^{a}$ Results for the ranking were skewed, therefore a Mann Whitney $U$ test was performed ${ }^{b}$ One-tailed

* Significant at $p<0.05$

\section{Table 2}

Results on the BRIT for the elite and non-elite badminton players (mean $\pm S D$ ) and analysis of variance results for comparisons of means between groups

\begin{tabular}{|c|c|c|c|c|c|}
\hline & \multirow[b]{2}{*}{ Elite } & \multirow[b]{2}{*}{ Non-elite } & \multicolumn{2}{|l|}{ ANOVA } & \multirow[b]{2}{*}{$d^{c}$} \\
\hline & & & $F(d f)$ & $p^{\mathrm{b}}$ & \\
\hline \multicolumn{6}{|l|}{ Reaction time } \\
\hline Domain-general & $\mathrm{n}=13$ & $\mathrm{n}=9$ & & & \\
\hline DGRT (ms) & $336 \pm 41$ & $346 \pm 56$ & $0.239(1,20)$ & 0.630 & 0.21 \\
\hline Badminton-specific & $\mathrm{n}=10$ & $\mathrm{n}=7$ & & & \\
\hline Go BSRT (ms) & $433 \pm 93$ & $538 \pm 64$ & $6.650(1,15)$ & $0.021^{*}$ & 1.27 \\
\hline Go forehand BSRT (ms) & $427 \pm 107$ & $487 \pm 71$ & $1.674(1,15)$ & 0.215 & 0.64 \\
\hline Go backhand BSRT (ms) & $438 \pm 100$ & $544 \pm 65$ & $6.082(1,15)$ & $0.026^{*}$ & 1.22 \\
\hline Go-stop BSRT (ms) & $455 \pm 92$ & $513 \pm 80$ & $1.811(1,15)$ & 0.198 & 0.66 \\
\hline Go-stop forehand BSRT (ms) & $451 \pm 94$ & $482 \pm 95$ & $0.465(1,15)$ & 0.506 & 0.34 \\
\hline Go-stop backhand BSRT (ms) & $460 \pm 94$ & $543 \pm 70$ & $3.955(1,15)$ & 0.065 & 0.98 \\
\hline \multicolumn{6}{|l|}{ Inhibitory control } \\
\hline Domain-general & $n=13$ & $\mathrm{n}=9$ & & & \\
\hline DGIC (ms) & $207 \pm 28$ & $209 \pm 17$ & $-0.205(1,20)$ & 0.840 & 0.09 \\
\hline Badminton-specific & $\mathrm{n}=10$ & $\mathrm{n}=7$ & & & \\
\hline $\mathrm{BSIC}(\mathrm{ms})$ & $235 \pm 83$ & $234 \pm 75$ & $0.001(1,15)$ & 0.981 & -0.01 \\
\hline
\end{tabular}

a Wilks Lambda; $b$ Two-tailed

$c$ Effect-size Cohen's $d,>0.2=$ small,$>0.5=$ medium, $>0.8=$ large, large effect-sizes are depicted in bold; * Significant at $p<0.05$ 
Table 3

Spearman's rho correlation coefficient for ranking vs. all test variables for the elite and non-elite separately

\begin{tabular}{|c|c|c|c|c|}
\hline & \multicolumn{2}{|l|}{ Elite } & \multicolumn{2}{|c|}{ Non-Elite } \\
\hline & Q & $\mathrm{R}^{2}(\%)$ & Q & $\mathrm{R}^{2}(\%)$ \\
\hline \multicolumn{5}{|l|}{ Reaction time } \\
\hline Domain-general & $\mathrm{n}=13$ & & $\mathrm{n}=9$ & \\
\hline DGRT (ms) & $0.70^{* *}$ & 50 & $0.70^{*}$ & 49 \\
\hline Badminton-specific & $\mathrm{n}=10$ & & $\mathrm{n}=7$ & \\
\hline Go BSRT (ms) & 0.32 & 10 & -0.07 & 1 \\
\hline Go forehand BSRT (ms) & 0.27 & 7 & -0.25 & 6 \\
\hline Go backhand BSRT (ms) & 0.27 & 7 & -0.57 & 33 \\
\hline Go-stop BSRT (ms) & 0.48 & 23 & -0.21 & 5 \\
\hline Go-stop forehand BSRT (ms) & 0.58 & 33 & -0.21 & 5 \\
\hline Go-stop backhand BSRT (ms) & 0.38 & 15 & -0.25 & 6 \\
\hline \multicolumn{5}{|l|}{ Inhibitory control } \\
\hline Domain-general & $\mathrm{n}=13$ & & $\mathrm{n}=9$ & \\
\hline DGIC (ms) & -0.27 & 7 & $<0.01$ & $<1$ \\
\hline Badminton-specific & $\mathrm{n}=10$ & & $\mathrm{n}=7$ & \\
\hline BSIC (ms) & 0.58 & 33 & $-0.89^{* *}$ & 80 \\
\hline
\end{tabular}

All tests were two-tailed

$\rho=$ Spearman's Rho; $R^{2}=$ Coefficient of determination, measure of effect size

$(>1=$ small, $>6=$ medium, $>15=$ large $)$, large effect-sizes are depicted in bold

* significant at $p<0.05 ;{ }^{* *}$ significant at $p<0.01$

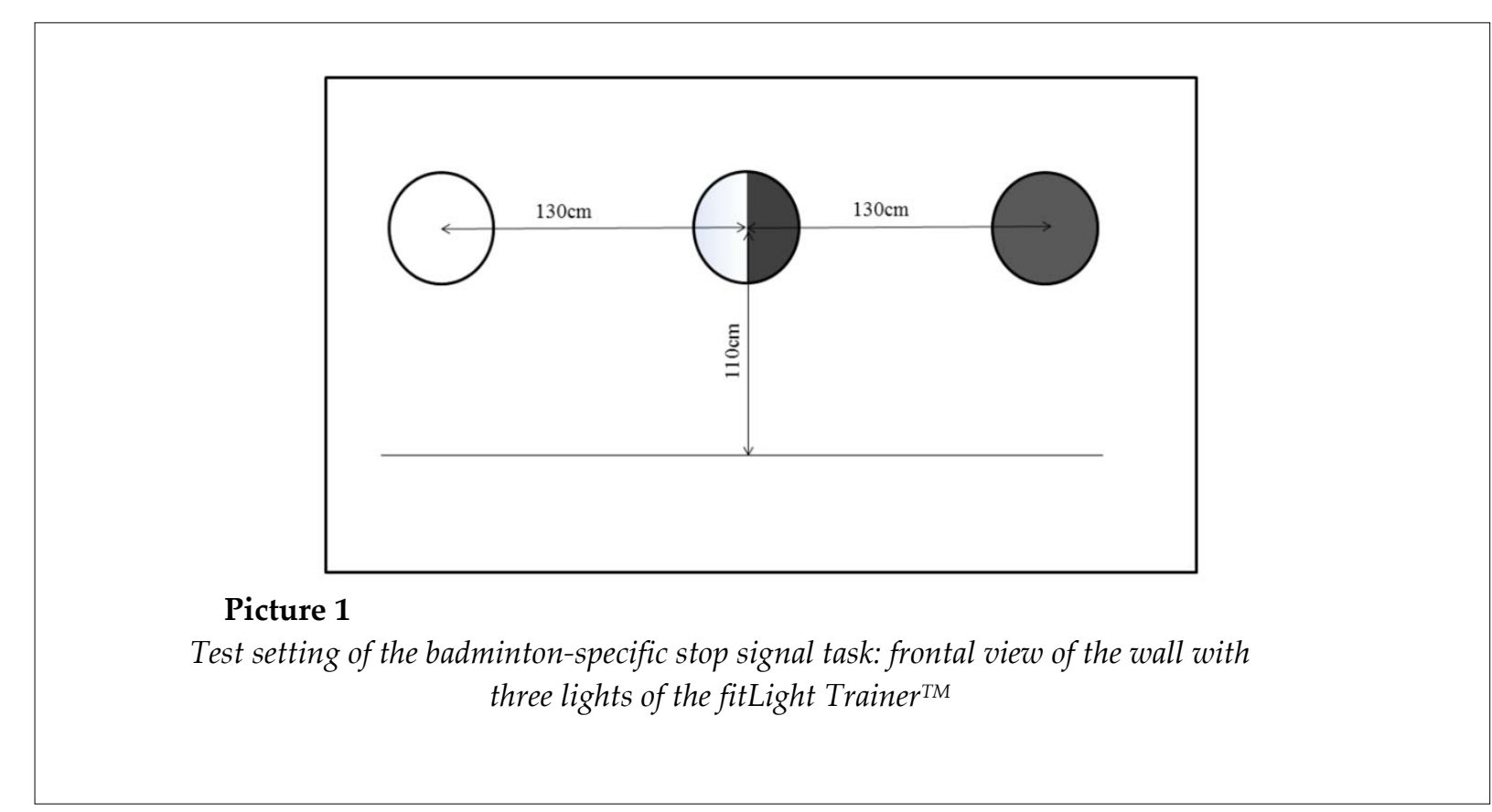




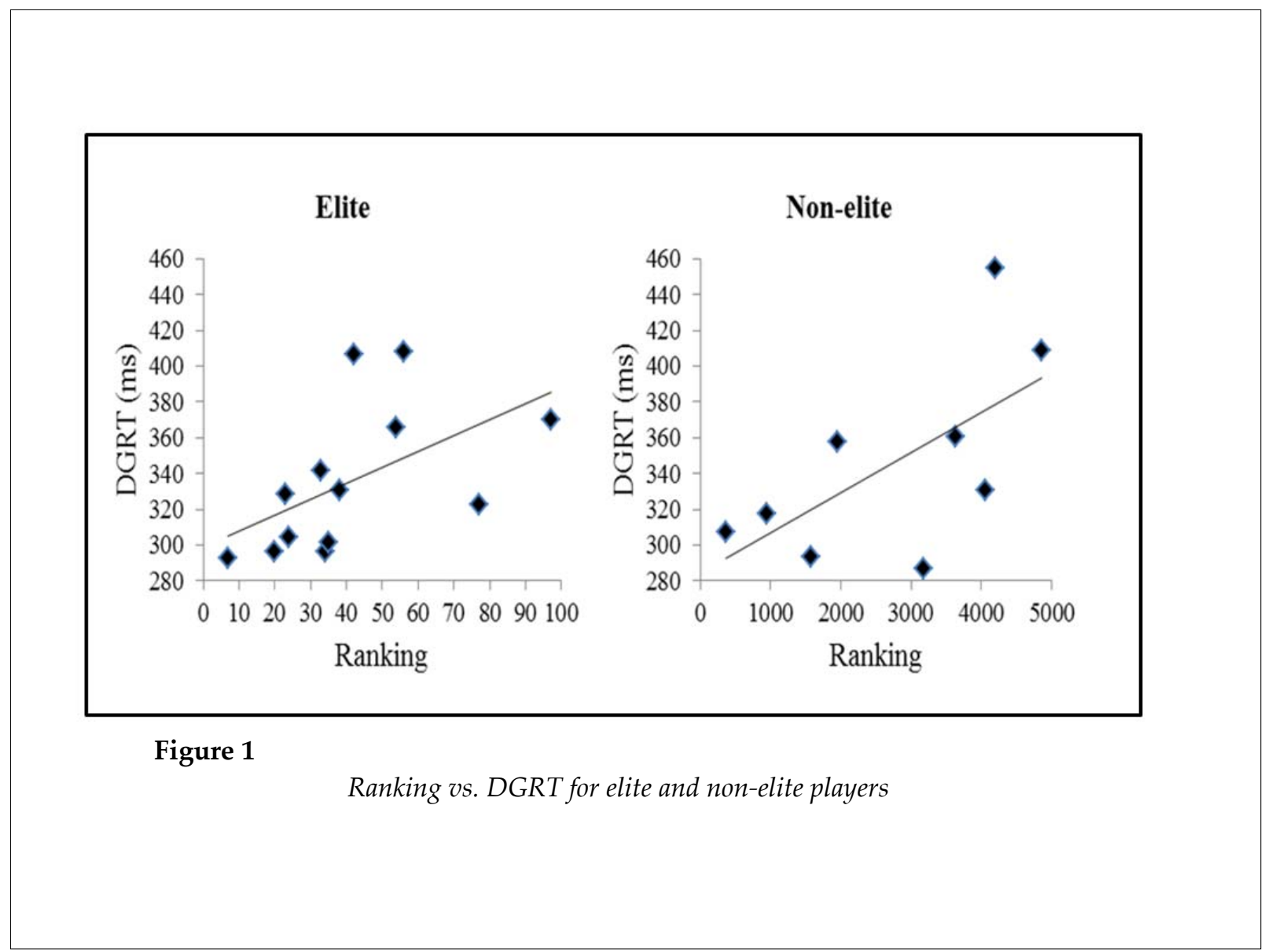

\section{Discussion}

This study evaluated the reproducibility and validity of the newly developed Badminton Reaction Inhibition Test (BRIT) for the assessment of domain-general and badminton-specific reaction time as well as inhibitory control in elite and non-elite badminton players. Acceptable reproducibility outcomes for badminton-specific reaction time and badminton-specific inhibitory control were presented. Good construct validity was shown for badminton-specific reaction time as it discriminated between elite and non-elite players. No differences between elite players and non-elite players were found in domain-general reaction time or in both components of inhibitory control. Concurrent validity for domain-general reaction time was good, as it was associated with the national ranking for both elite and non-elite players. No relationship was found between the national ranking and badminton-specific reaction time or both components of inhibitory control.

The domain-general stop signal task was used for the assessment of domain-general cognitive performance (Logan and Cowan, 1984). The difference between the elite and non-elite group in domain-general reaction time of $10 \mathrm{~ms}$ was not statistically significant. However, in elite badminton match play average shuttle velocity lies around $19 \mathrm{~m} / \mathrm{s}$ (Phomsoupha and Laffaye, 2014, 2015), meaning that the shuttle travels $19 \mathrm{~cm}$ in $10 \mathrm{~ms}$. This could make the difference between a perfect or a poor return of the shuttle. A significant relationship between domain-general reaction time and the national ranking was revealed, which indicates the need of a fast reaction time for elite performance. This finding does underscore the validity of the domaingeneral stop signal task for the assessment of domain-general reaction time in badminton players.

The BRIT was assumed to be valid for the assessment of badminton-specific reaction time as elite players were found to outscore non-elite players. However, this was only true for measures of badminton-specific (backhand) reaction time, but not for badminton-specific forehand reaction 
time. The difference between the assessment of badminton-specific backhand and forehand reaction time was the direction in which the player had to move to deactivate the outer light. In addition, players had to cover extra distance during the assessment of backhand reaction time as the outer light was positioned further away from the preferred hand than during the assessment of forehand reaction time. These findings question the validity of the badmintonspecific component of the BRIT as a measure of solely reaction time and suggest that the test results are partly determined by movement ability, especially to the backhand side. Importance of movement ability in elite badminton is supported by research of Madsen et al. (2015) who showed that elite badminton players outscored sub-elite players in a badminton-specific movement speed test. Since both reaction time and movement ability seem important for elite badminton performance, the assessment of badminton-specific reaction time of the BRIT can still be called a valid measure as it discriminates between elite and non-elite players with regard to characteristics important for badminton match play.

Reproducibility of the domain-general stop signal task used for the assessment of domain-general inhibitory control had been already confirmed (Congdon et al., 2012; Williams et al., 1999). The current study presented poor to fair relative reliability for the assessment of badminton-specific inhibitory control by means of the badminton-specific stop signal task. However, relative reliability is strongly dependent on sample size and as the current study included only five test-retest measures, future research including a larger sample size is needed to provide an accurate view on the relative reliability of the badminton-specific stop signal task (Weir, 2005). Measures of agreement showed a reasonable coefficient of variation of $13 \%$ for the assessment of badminton-specific inhibitory control. This is in agreement with the coefficient of variation ranging from 3 to $19 \%$ presented in reproducibility studies on the assessment of perceptuo-motor skills (Faber et al., 2014, 2015).

No differences between elite and non-elite players were revealed in domain-general and badminton-specific inhibitory control. A possible explanation is that the BRIT was not able to detect existing differences. Another explanation for the finding that elite and non-elite players did not significantly differ in inhibitory control could be that the participant group was too homogeneous. The current study included elite Dutch badminton players. It can be doubted whether the included participants really reflected badminton elite performance as the players had a ranking higher than 1000 on the Badminton World Federation Men's Singles Ranking. To achieve elite performance in badminton, one must excel in different dimensions (Elferink-Gemser et al., 2011). Perhaps in the current study the elite players outscored the non-elite on tactical or physical measures, but not on cognitive performance. However, at badminton's world elite level a player must excel in multiple domains and compensation of a less developed domain seems not possible. Therefore, future research is recommended to include world class elite players to give an accurate evaluation of the need of inhibitory control in badminton elite performance.

The BRIT gives a valid indication of reaction time and could be used by coaches and players for monitoring training progress and players' development, it may also provide input for training programs aiming to improve players' badminton performance. Furthermore, it can be applied to address questions whether a highly developed reaction time is innate, causing that the players with better innate reaction abilities have a higher chance to achieve elite badminton performance, or whether elite players have a faster reaction time due to their badminton experience, which means that a faster reaction time can be developed by training and match play. A review of Smith et al. (2010) who examined the effects of aerobic exercise on neurocognitive performance in healthy adults presented a faster reaction time in subjects that had followed aerobic interventions of more than one month duration. Although this provides evidence for trainability of reaction time, literature on the topic is still scarce. Future research should monitor the development of reaction time in talented badminton players longitudinally to find out if the development of reaction time of the players who finally achieve elite performance differs from that of non-elite players. Insight into the development of reaction time could help coaches in the process of talent 
identification and development by designing specific training programs.

The present study has several limitations. One of them is the small number of participants in the reproducibility part of the study. Future research including a larger sample size and world elite players should be conducted to get a better view on the reproducibility and validity for the assessment of inhibitory control. Furthermore, the validity and reproducibility of the BRIT were only assessed in a population of adults. It is recommended to perform a similar study in children of different age and performance levels to examine the value of the BRIT for monitoring badminton performance in different age groups.
The current study focused on the evaluation of the reproducibility and validity of a newly developed test for the assessment of domain-general and badminton-specific reaction time as well as inhibitory control in badminton players. In conclusion, reproducibility and validity of inhibitory control assessment were not confirmed, however, the BRIT appears a reproducible and valid measure of reaction time in badminton players. Reaction time measured with the BRIT may provide input for training programs aiming to improve badminton players' performance.

\section{Acknowledgements}

The authors would like to thank all participants for their cooperation and the students of Human Movement Sciences for their help with testing. Special thanks go to Sven Müller and Nikki Kolman for their assistance during test development and testing, to Joris van Soerland of fitLight Benelux for providing the fitLight TrainerTM and to Badminton Nederland for the support in promoting the research and giving us the opportunity to recruit and assess players during the Dutch national badminton championships.

\section{References}

Alves H, Voss MW, Boot WR, Deslandes A, Cossich V, Salles JI, Kramer AF. Perceptual-cognitive expertise in elite volleyball players. Front Psychol, 2013; 4: 1-9

Congdon E, Mumford JA, Cohen JR, Galvan A, Canli T, Poldrack RA. Measurement and reliability of response inhibition. Front Psychol, 2012; 3: 1-10

Diamond A. The early development of executive functions. Lifespan Cognition: Mechanisms of Change. Oxford University Press, 70-95; 2006

Elferink-Gemser MT, Jordet G, Coelho-E-Silva MJ, Visscher C. The marvels of elite sports: how to get there? Brit J Sport Med, 2011; 45: 683-684

Faber IR, Bustin PMJ, Oosterveld FGJ, Elferink-Gemser MT, Nijhuis-Van der Sanden MWG. Assessing personal talent determinants in young racquet sport players: a systematic review. J Sports Sci, 2016; 34: 395-410

Faber IR, Nijhuis-Van Der Sanden MWG, Elferink-Gemser MT, Oosterveld FGJ. The Dutch motor skills assessment as tool for talent development in table tennis: a reproducibility and validity study. J Sports Sci, 2015; 33: 1149-1158

Faber IR, Elferink-Gemser MT, Oosterveld FGJ, Nijhuis-Van der Sanden MWG. Revision of two test items of the dutch motor skills assessment measuring ball control in young table tennis players: a reproducibility and validity study. Ann Res SportPhys Act, 2014: 53-69

Fleiss JL. The Design and Analysis of Clinical Experiments. Hoboken: John Wiley \& Sons, Inc; 1999

Hong Y, Wang SJ, Lam WK, Cheung JTM. Kinetics of badminton lunges in four directions. J Appl Biomech, 2014; 30: 113-118

Huijgen BCH, Leemhuis S, Kok N, Verburgh, L, Oosterlaan, J, Elferink-Gemser, MT, Visscher, C. Cognitive functions in elite and sub-elite youth soccer players aged 13 to 17 years. PloS One, 2015; 10: e0144580

Kida N, Oda S, Matsumura M. Intensive baseball practice improves the go/nogo reaction time, but not the 
simple reaction time. Cognitive Brain Res, 2005; 22: 257-264

Kuntze G, Mansfield N, Sellers WA. Biomechanical analysis of common lunge tasks in badminton. J Sport Sci, 2010; 28: 183-191

Lees A. Science and the major racket sports: a review. J Sport Sci, 2003; 21: 707-732

Logan GD, Cowan WB. On the ability to inhibit thought and action: a theory of an act of control. Psychol Rev, 1984; 91: 295-327

Loureiro LFB, Freitas PB. Influence of the performance level of badminton players in neuromoter aspects during a target-pointing task. Rev Bras Med Esporte, 2012; 18: 203-207

Madsen CM, Karlsen A, Nybo L. Novel speed test for evaluation of badminton-specific movements. J Strength Cond Res, 2015; 29: 1203-1210

Mann D, Williams A, Ward P, Janelle C. Perceptual-cognitive expertise in sport: a meta-analysis. J Sport Exercise Psy, 2007; 29: 457-464

Phomsoupha M, Laffaye G. Shuttlecock velocity during a smash stroke in badminton evolves linearly with skill level. Comput Meth Biomechanics and Biomed Eng, 2014; 17: 140-141

Phomsoupha M, Laffaye G. The science of badminton: game characteristics, anthropometry, physiology, visual fitness and biomechanics. Sports Med, 2015; 45: 473-495

Smith PJ, Blumenthal JA, Hoffman BM, Cooper H, Strauman TA, Welsh-Bohmer K, Browndyke JN, Sherwood A. Aerobic exercise and neurocognitive performance: a meta-analytic review of randomized controlled trials. Psychosom Medicine, 2010; 72: 239-252

Verbruggen F, Logan GD. Models of response inhibition in the stop-signal and stop-change paradigms. Neurosci Biobehav R, 2009; 33: 647-61

Verburgh L, Scherder EJA, van Lange PAM, Oosterlaan J. Executive functioning in highly talented soccer players. PloS One, 2014; 9: e91254

Vestberg T, Gustafson R, Maurex L, Ingvar M, Petrovic P. Executive functions predict the success of topsoccer players. PloS One, 2012; 7: e34731

de Vet HCW, Terwee CB, Knol DL, Bouter LM. When to use agreement versus reliability measures. J Clin Epidemiol, 2006; 59: 1033-1039

Voss MW, Kramer AF, Basak C, Prakash RS, Roberts B. Are expert athletes "expert" in the cognitive laboratory? A meta-analytic review of cognition and sport expertise. Appl Cognitive Psych, 2010; 24: $812-826$

Weir J. Quantifying test-retest reliability using the intraclass correlation coefficient and the SEM. J Strength Cond Res, 2005; 19: 231-240

Wetherell A. Cognitive and psychomotor performance tests and experiment design in multiple chemical sensitivity. Environ Health Persp, 1997; 105: 495-503

Williams BR, Ponesse JS, Schachar RJ, Logan GD, Tannock R. Development of inhibitory control across the life span. Dev Psychol, 1999; 35: 205-213

\section{Corresponding author:}

\section{Barbara Huijgen}

Address: Center for Human Movement Sciences, University Medical Center Groningen, University of Groningen, Antonius Deusinglaan 1, 9713 AV Groningen. Netherlands

Phone: + 31/06/25646582

Fax: $+31 / 50 / 3633150$

E-mail: b.c.h.huijgen@umcg.nl 